\title{
Level and length of cyclic solar activity during the Maunder minimum as deduced from the active-day statistics
}

\author{
J. M. Vaquero ${ }^{1}$, G. A. Kovaltsov ${ }^{2}$, I. G. Usoskin ${ }^{3}$, V. M. S. Carrasco ${ }^{4}$, and M. C. Gallego ${ }^{4}$ \\ ${ }^{1}$ Departamento de Física, Universidad de Extremadura, 06800 Mérida, Spain \\ 2 Ioffe Physical-Technical Institute, 194021 St. Petersburg, Russia \\ 3 Sodankylä Geophysical Observatory and ReSoLVE Center of Excellence, University of Oulu, 90014 Oulu, Finland \\ e-mail: Ilya.Usoskin@oulu.fi \\ ${ }^{4}$ Departamento de Física, Universidad de Extremadura, 06071 Badajoz, Spain
}

Received 25 February 2015 / Accepted 25 March 2015

\section{ABSTRACT}

\begin{abstract}
Aims. The Maunder minimum (MM) of greatly reduced solar activity took place in $1645-1715$, but the exact level of sunspot activity is uncertain because it is based, to a large extent, on historical generic statements of the absence of spots on the Sun. Using a conservative approach, we aim to assess the level and length of solar cycle during the MM on the basis of direct historical records by astronomers of that time.

Methods. A database of the active and inactive days (days with and without recorded sunspots on the solar disc) is constructed for three models of different levels of conservatism (loose, optimum, and strict models) regarding generic no-spot records. We used the active day fraction to estimate the group sunspot number during the MM.

Results. A clear cyclic variability is found throughout the MM with peaks at around 1655-1657, 1675, 1684, 1705, and possibly 1666 , with the active-day fraction not exceeding $0.2,0.3$, or 0.4 during the core MM, for the three models. Estimated sunspot numbers are found to be very low in accordance with a grand minimum of solar activity.

Conclusions. For the core MM (1650-1700), we have found that (1) A large portion of no-spot records, which correspond to the solar meridian observations, may be unreliable in the conventional database. (2) The active-day fraction remained low (below 0.3-0.4) throughout the MM, indicating the low level of sunspot activity. (3) The solar cycle appears clearly during the core MM. (4) The length of the solar cycle during the core MM appears for $9 \pm 1$ years, but this is uncertain. (5) The magnitude of the sunspot cycle during MM is assessed to be below 5-10 in sunspot numbers. A hypothesis of the high solar cycles during the MM is not confirmed.
\end{abstract}

Key words. Sun: activity - sunspots

\section{Introduction}

There was a period in the second part of the 17th century of greatly reduced solar activity that was named the Maunder minimum (MM) by Eddy (1976). The MM was characterized by an almost complete absence of reported sunspots on the solar surface, although some indications of cyclic activity can be noticed, particularly in the geomagnetic and heliospheric indices (Beer et al. 1998; Usoskin et al. 2001; Soon \& Yaskell 2003). The reconstruction of solar activity based on the historical records of telescopic observations of sunspots since 1610 (Hoyt \& Schatten 1998a,b - called HS98 henceforth) marked a milestone in the study of solar activity in the recent past and, especially, for the MM period. The group sunspot number (GSN) built by HS98 became the only high-resolution (daily) index to study solar activity during the MM.

The aim of this work is to elucidate whether the absence of the cyclic sunspot activity during the MM was real or an artefact caused by a problem in the compilation of the database of sunspot records. Several studies pointed to possible inconsistences in the database used by HS98, especially around the MM (e.g., Vaquero \& Vázquez 2009; Vaquero et al. 2011; Vaquero \& Trigo 2014). As an extreme, Zolotova \& Ponyavin (2015) claim there was no grand $\mathrm{MM}$ and that sunspot cycles during MM were as high as $\approx 100$, which is higher than the current cycle \# 24 . We note that the MM is covered well by sunspot data, and more than
$90 \%$ of the days have formal observation records in the HS98 database. However, it contains a large number of generic statements of the absence of sunspots during a long period of time.

Such records are not strict observational data, but they were interpreted by HS98 as no-spot data. Many of these records corresponded to solar meridian observations (Vaquero 2007; Clette et al. 2014) and should be used with caution for the reconstruction of solar activity, as shown by Vaquero et al. (2014), who analysed sunspot records taken during systematic solar meridian observations performed at the Royal Observatory of the Spanish Navy from 1833 to 1840 . Moreover, as Carrasco et al. (2015) suggest, based on an analysis of sunspot records by Hevelius in the 17th century, the GSN index may be underestimated during the MM owing to a large number of "zero" sunspot records taken from solar meridian observations.

In general, astrometric observations of the Sun are not always reliable for sunspot counting because of the different aim of such observations. For example, there is no information on sunspots in the extensive table of astrometric records of the Sun made with the meridian line in the San Petronio Basilica from 1645 to 1735 as published by Manfredi (1736). Nevertheless, Hoyt \& Schatten (1998a) adopted solar observations recorded in this source as no-spot reports, which is not correct. It has been discussed that, while the definition of sunspot numbers and even sunspot groups is not very reliable in the earlier part of the GSN 
series (Clette et al. 2014; Zolotova \& Ponyavin 2015), solar activity during the MM can be reliably represented by the fraction of active days (Kovaltsov et al. 2004; Vaquero et al. 2012, 2014; Usoskin 2013).

Despite the overall level of activity, the parameters of the solar cyclic variability during MM are also important to know. Although the solar cycle was perceptible in the butterfly diagram (Ribes \& Nesme-Ribes 1993; Vaquero et al. 2015) based on the observations of sunspot latitudes during the last decades of the 17th century, the 11-year solar cycle is only marginally detectable in the sunspot numbers (Waldmeier 1961; Mendoza 1997) with a dominat weak 22-year cycle (Usoskin et al. 2001). On the other hand, some works based on data for high-resolution cosmogenic ${ }^{14} \mathrm{C}$ measured in tree trunks suggest that the solar cycle might have been stretched during Grand solar minima (Stuiver et al. 1998; Miyahara et al. 2004, 2006, 2010; Nagaya et al. 2012; Miyake et al. 2013). These studies have suggested that the length of the solar cycle was increased to about 14 years during the MM, to about 13 years in the beginning of the Spörer minimum, up to 16 years during the fourth-century BC minimum, and 12-13 years during the late seventh-century minima.

In this work we aim to study the variability of solar activity during the MM using the statistics of the active days basing on only the most reliable solar observations from the database compiled by Hoyt \& Schatten (1998a) and to establish an uppermost upper (maximum maximorum) limit on that.

\section{Sunspot activity database}

Since quantitative interpretation of many records is uncertain for that period, we consider only qualitative indicators of the sunspot activity for each day for the period 1637-1715 AD. Leaving aside the exact number of reported sunspot group in the HS98 catalogue, we only consider three possible states for each day:

- no-information or missing days;

- inactive days when we believe there were reliable observation of the absence of sunspots;

- active days when at least one sunspot group was explicitly reported by at least one observer.

We built our database of the active and inactive days for three models of different levels of conservatism regarding generic nospot records. For the period 1637-1642, we used exactly the records listed by Vaquero et al. (2011). For the period 16431715, we used the records from the HS98 database ${ }^{1}$ for each observer separately. In addition, for the year 1672, several active days were added according to observations by N. Bion that were not included in the HS98 database (Casas et al. 2006). While the original HS98 database contains 26508 daily records for the analysed period 1637-1715, our models include fewer records because of rejecting, with different levels of conservatism, generic statements that were mostly related to no-spot observations. All these models provide an overestimated upper bound of sunspot activity owing to a possible selection bias towards active days.

\subsection{Loose model (ML)}

This model is similar to the one by Kovaltsov et al. (2004), and it ignores all the generic statements (longer than a month) in the

\footnotetext{
1 http://wWw.ngdc.noaa.gov/stp/sunspot_numbers/group_ sunspot_numbers/alldata.dat
}

HS98 database, and considers only explicit statements that mention exact dates of observations. This affects such generic statements as those by J. Hevelius for no spots during 1645-1651, by J. Picard for 1653-1665, and by H. Siverius for 1675-1689. This model is the least conservative and is called "loose". It includes 13512 observational days, which is nearly half of the HS98 database.

\subsection{Optimum model (MO)}

The MO model provides a reasonable balance between strictness and data acceptance and is considered as the optimum conservative mode. For each year, we considered observations of only those observers who reported at least one sunspot group at any day of the year, which would prove that the observer was "active". In this way, extended generic reports of "no-spot" were neglected, but no-spot records of active observers were considered. The MO models is biased towards "active" years and produces no result for the years without sunspot observations. For example, if a year is full of definite "no-spot" records but does not contain a single reported sunspot observation, this year is marked as "no-information" in this model. Alternatively, if an observer was "active" during a year, his generic "no-spot" records for this year were considered by the model, so that the total number of days $N_{\mathrm{T}}$ in the MO model may exceed that for the ML model for several years (see Fig. 1). This model includes 8089 observational days for the period analysed, which is roughly one third of the full HS98 database.

\subsection{Strict model (MS)}

In this "strict" model we excluded all the generic statements as in the ML models, but additionally treated other no-spot records in a very conservative way, so that we consider as inactive only those days when at least two observers independently reported that the Sun was spotless and when there were no other records of sunspots. If at least one observer reported sunspots, the day was considered as active. All other days were treated as noinformation days. This is the most conservative approach, especially in the earlier part of the Maunder minimum, when the number of documented observers was low and they rarely overlap. This model includes 5159 daily records or one fifth of the full HS98 database.

For each model we define the number of active $N_{\mathrm{A}}$ and the total number $N_{\mathrm{T}}$ of the accepted observational days in a year. Since the annual data are quite noisy (see below), we also consider triennial intervals. To keep this strictness, the MO model was still operating with annual periods to identify "active" observers. The results are shown in Fig. 1 for the three models, as well as for the formal HS98 database. One can see that, while the HS98 database covers the entire period fairly well, the three models provide a more conservative estimate of reliable observations, which is greatly reduced in the earlier MM but quite solid towards its end.

An example of the coverage of the data in the three models and the formal HS98 database is shown in Fig. 2 for the year 1676. Although this year was almost fully covered by data in the formal HS98 database, except for a short gap in October, the three models considered here include fewer inactive days while keeping the active days. A small discrepancy in the number of active days is related to excluded interpolations (as in Dec. 22-24) and confusing values (as in Jun. 25 when a sunspot 
J. M. Vaquero et al.: Solar activity during the Maunder minimum

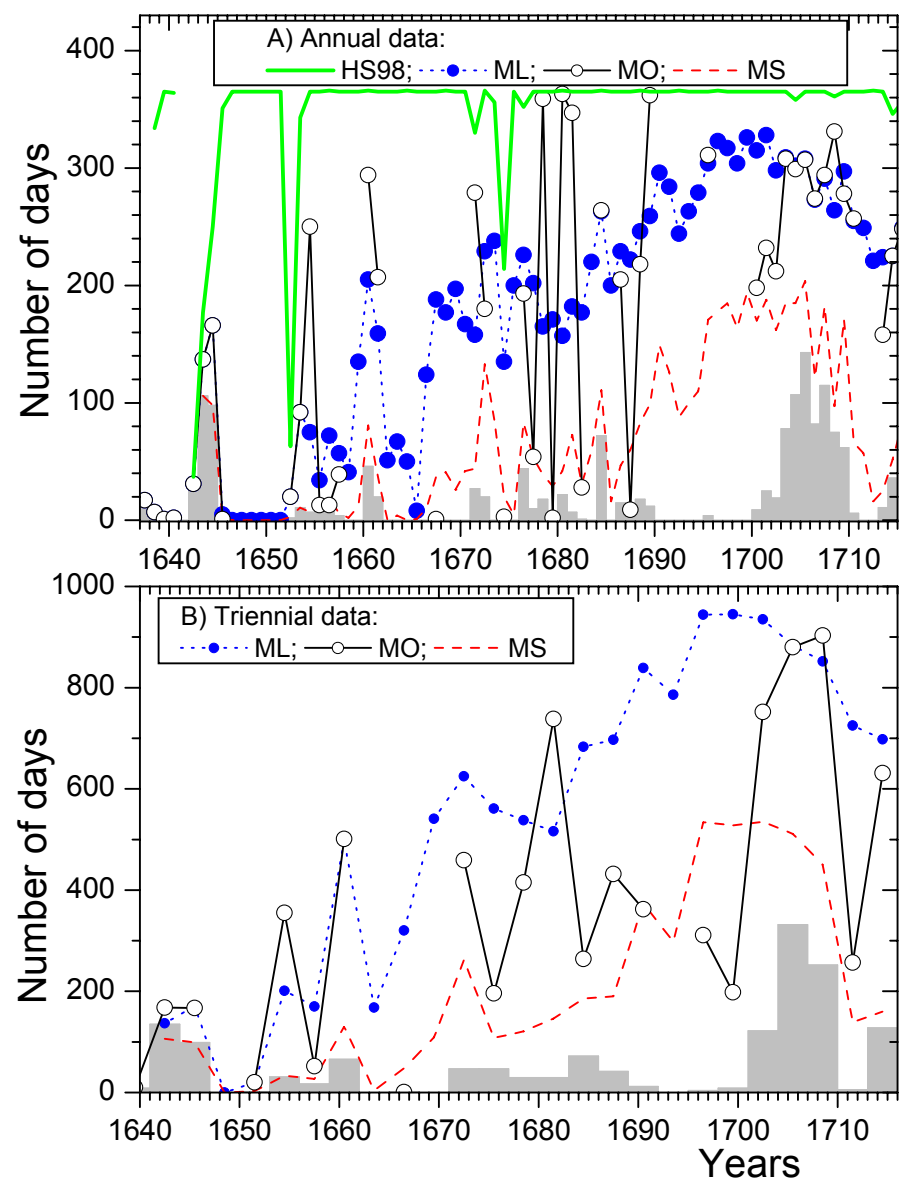

Fig. 1. Number of active days $N_{\mathrm{A}}$ (grey bars) and total observational days $N_{\mathrm{T}}$ (curves as defined in the legend for the three models and the formal HS98 database) per interval used. Panels A and B depict annual and triennial data, respectively.

record by R. Hook was missed in the formal HS98 series) in the HS98 database.

We emphasize again that the procedure described above serves as the highest upper bound because of possible oversuppressing zero-sunspot records.

\section{Results}

\subsection{Active day fraction}

From the collected database of sunspot records, we have estimated the fraction of active days $F_{\mathrm{A}}$ in each model, as follows (cf. Kovaltsov et al. 2004). For each interval, either annual or triennial, we have a sample of $n$ daily observations with $r$ active days reported. Assuming these observation were taken randomly and independently, one can assess the probability of the occurrence of exactly $s$ active days within $N$ days during the considered interval (1 year or 3 years) using the hypergeometric probability distribution:

$$
p(s)=\frac{s !(N-s) !}{(s-r) !(N-s-n+r) !} \cdot \frac{n !(N-n) !}{(n-r) ! N ! r !} .
$$

As the optimum value of $s *$, we consider the median value, that is to say, the value of $s$ that yields $P(s *) \equiv \sum_{r}^{s *} p(s)=0.5$. The results for annual and triennial time intervals are shown, along with error bars of a $90 \%$ (two-sided) confidence interval, in Figs. 3 and 4, respectively. We note that triennial data were

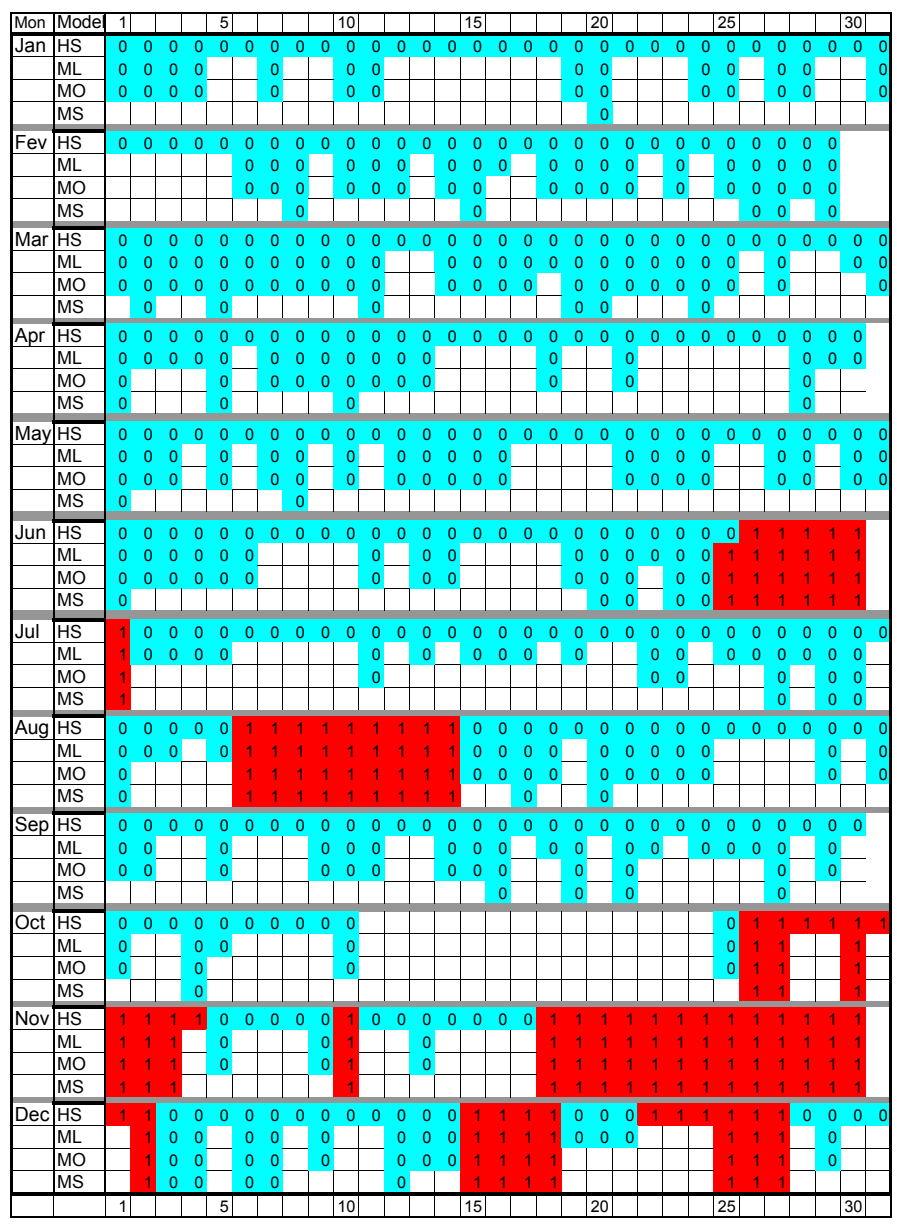

Fig. 2. Map of the days allocated for the year 1676 in the original HS98 database (denoted as HS) and the three models considered here. Each line represents one month (days of the month numbered on the top and bottom) for a model. The empty white, blue " 0 ", and red " 1 " cells correspond to no-information, no-spot, and active days.

calculated from the original daily values using Eq. (1) and not as an average of the annual data.

\subsection{Length of solar cycles}

Although the annual data are quite noisy, the triennial ones (see Table 1) clearly show a decadal periodicity during the MM. For example, Fig. 4 suggests maxima of solar cycles around 1639, $1655-1657,1675,1684$, and 1705 in all the models. There is also an indication of a cycle maximum around 1666 in the MO models, but the statistics are low with a single observation for the three-year interval. Periods around 1648 and 1693 are poorly known with data gaps in the MO model.

There are four solar activity maxima in the core MM, between maxima in around 1657 and 1684. This leads to an estimate of the average solar cycle length (max-to-max) during the core $\mathrm{MM}$ as $9 \pm 1$ years. However, our view of the cyclic evolution of sunspot activity during $\mathrm{MM}$ is uncertain because of the unclear situation around 1648, 1666, and 1693. If we assume two hypothetical missing solar maxima during these periods; for example, Waldmeier (1961) proposed a cycle maximum in 1649, while Usoskin et al. (2001) suggested a maximum around 1695, so we can estimate an average solar cycle length around the MM (from 1636 to 1711 ) to be $9.5 \pm 0.5$ years. If however, we assume that there were no additional solar cycle maxima around 


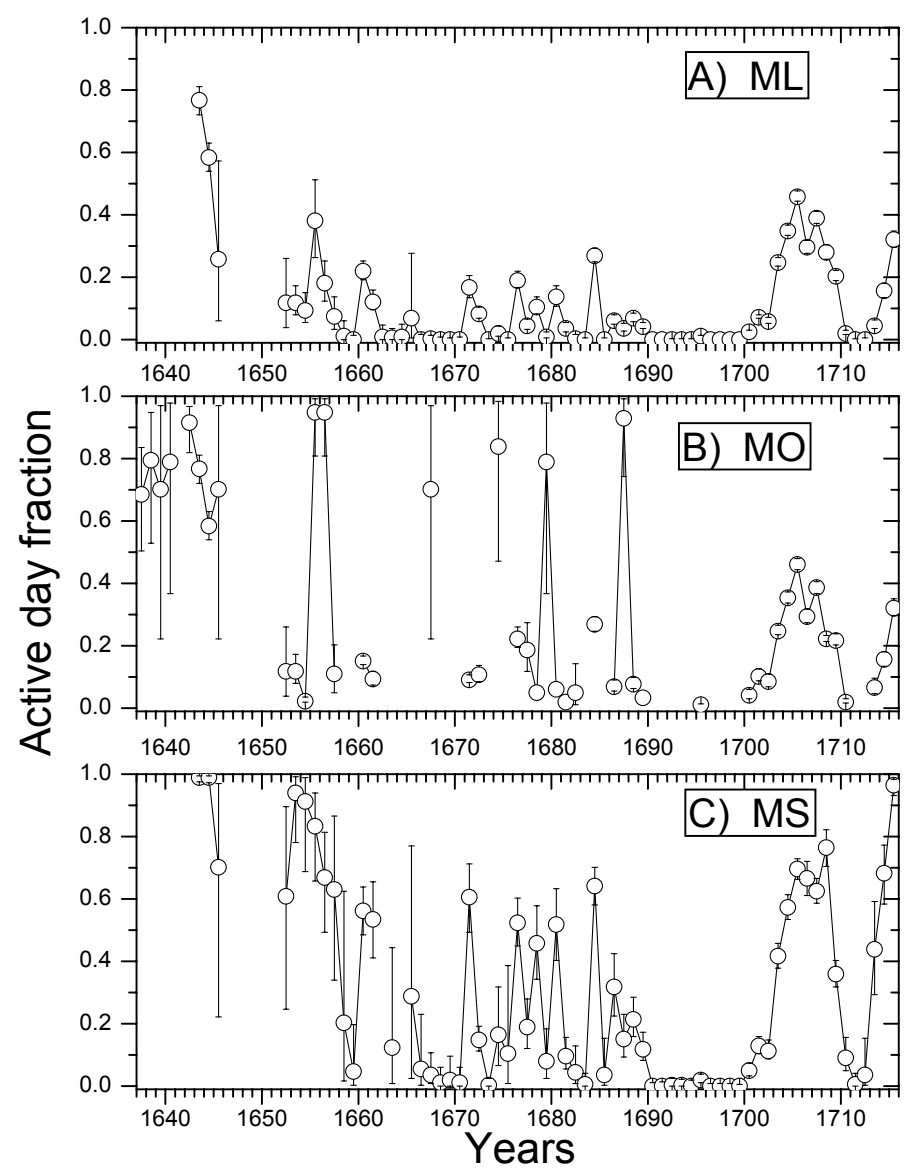

Fig. 3. Annual active day fraction for the three models. Error bars represent the $90 \%$ two-sided uncertainties.

1648 and 1693, the average cycle length (max-to-max) would be $13.2 \pm 0.6$ years. In this case, however, the length of individual cycles varies greatly, between 9 and 18 years. The estimated cycle length is similar to but somewhat shorter than the results proposed by Mendoza (1997) and Usoskin et al. (2001), who suggested the cycle length of 10.5-11 years during the MM using sunspot observations. Meanwhile, clustering of activity in $\approx 20$-year intervals $(1650-1670,1670-1690$, and 1690-1710) is also visible, in agreement with earlier results of the dominant 22-year periodicity during the MM (Usoskin et al. 2001). This clustering of activity, however, could also be produced because of the scarcity of reliable data around 1648, 1669, and 1693.

On the other hand, estimates of the cycle length based on cosmogenic ${ }^{14} \mathrm{C}$ data suggest much longer cycles during Grand minima (13-16 years). We note, however, that ${ }^{14} \mathrm{C}$ data cannot resolve individual cycles, because of the global carbon cycle attenuating high-frequency variability (Roth \& Joos 2013), but instead yields the mean periodicity over the interval analysed (e.g. Miyahara et al. 2004). This seeming contradiction between the results obtained here (see Mendoza 1997; Usoskin et al. 2001) and from ${ }^{14} \mathrm{C}$ data can be potentially reconciled in a view of the possible inversion of the cycle phase in the cosmic ray modulation during the periods of very weak activity like the MM (Owens et al. 2012). Thus, one or two cycles can be lost in the ${ }^{14} \mathrm{C}$ data, owing to forward and then reverse phase shifts in the beginning and end of the MM, leading to seemingly extended cycles in ${ }^{14} \mathrm{C}$ data.
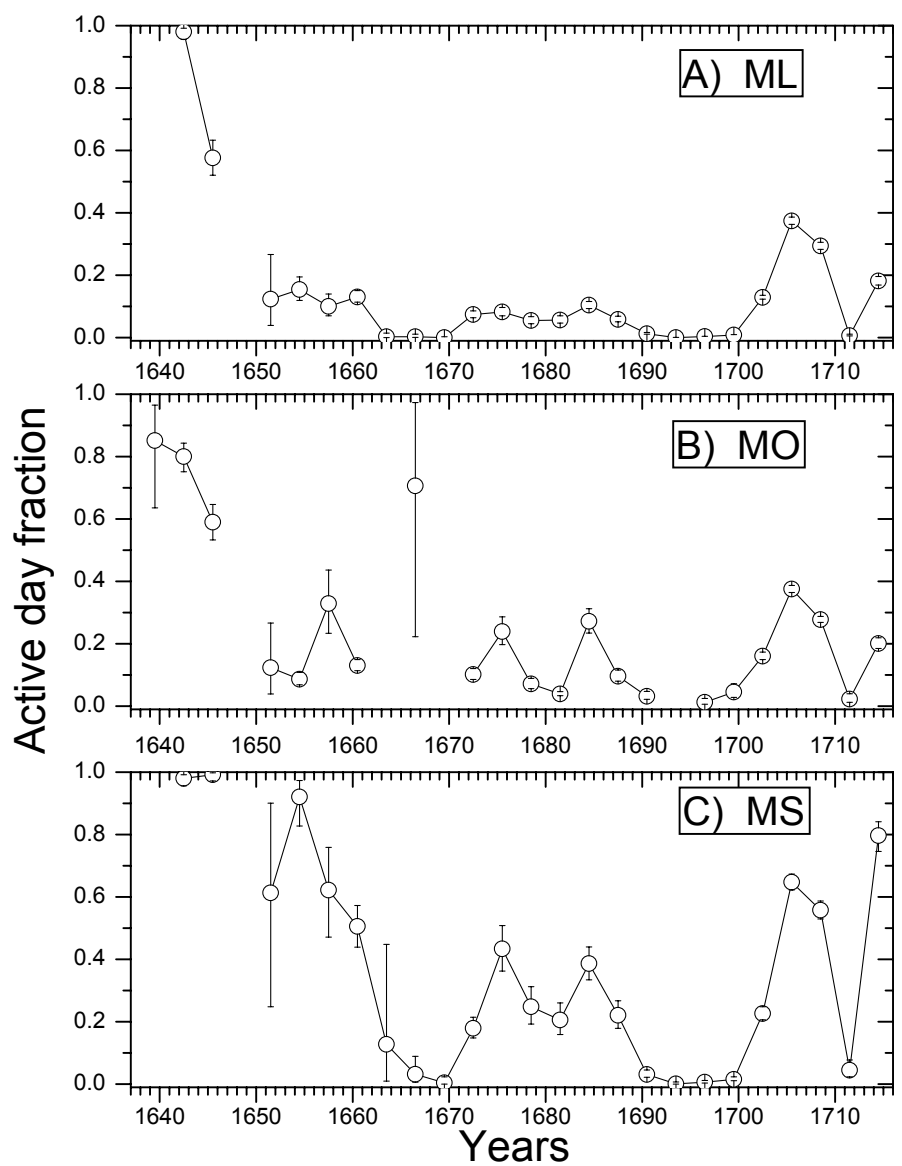

Fig. 4. Triennial active-day fraction for the three models. Error bars represent the $90 \%$ two-sided uncertainties. Digital data is available in Table 1.

\subsection{Sunspot numbers}

On average, the fraction of active days observed during MM was low, below 0.4 in the triennial data (Fig. 4) for ML and MO models, except for the year 1666 (MO model), which is however based on a single observation, and reaching up to $>0.7$ in the most conservative MS model. We note that, for the normal cycles, the active-day fraction is about $100 \%$ except for the years around solar minimum (Kovaltsov et al. 2004; Vaquero et al. 2012, 2014). The value of $F_{\mathrm{A}}$ was never below 0.15 for annual and 0.29 for triennial (see Fig. 5) during the period 1850 1995. Accordingly, such low values $F_{\mathrm{A}}$ even for the peaks during the MM correspond to (or are lower than) the minimum state of modern solar cycles. Therefore, although a cyclic activity during the MM is clear, at least during the core period, the sunspot cycles were weak, with the maxima being comparable to the modern cycle minima. We note that high solar cycles of the magnitude $40-100$ in sunspot number as proposed by Zolotova \& Ponyavin (2015) would unavoidably imply $\approx 100 \%$ active-day fraction (Vaquero et al. 2014) during most of the years, which contradicts the data (cf. Fig. 2).

To assess the sunspot number $R$ from the active-day fraction $F_{\text {A }}$, we apply a method developed by Kovaltsov et al. (2004) and Vaquero et al. $(2012,2014)$. For the annual data the relation was $R=19 \cdot F_{A}^{1.25}$ for $F_{A} \leq 0.5$ and $R=2.1 \cdot \exp \left(2.69 \cdot F_{\mathrm{A}}\right)$ for $0.5<F_{\mathrm{A}} \leq 0.8$ (Kovaltsov et al. 2004). The relation between triennial values $R$ and $F_{A}$ is shown in Fig. 5 for the period 18501995. One can see that the relation is quite good for $F_{\mathrm{A}}<0.8$ (with the only outlier related to the period 1954-1956, which 
Table 1. Triennial statistics of sunspot day occurrence for the three models considered here (see text for definition).

\begin{tabular}{|c|c|c|c|c|c|c|c|c|c|c|c|c|c|}
\hline \multirow[b]{2}{*}{ Year } & \multirow[b]{2}{*}{$N_{\mathrm{A}}$} & \multicolumn{4}{|c|}{ ML } & \multicolumn{4}{|c|}{$\mathrm{MO}$} & \multicolumn{4}{|c|}{ MS } \\
\hline & & $N_{\mathrm{T}}$ & $F_{\text {low }}$ & $F_{\text {med }}$ & $F_{\text {up }}$ & $N_{\mathrm{T}}$ & $F_{\text {low }}$ & $F_{\text {med }}$ & $F_{\text {up }}$ & $N_{\mathrm{T}}$ & $F_{\text {low }}$ & $F_{\text {med }}$ & $F_{\text {up }}$ \\
\hline 1639 & 9 & 10 & 0.636 & 0.851 & 0.965 & 10 & 0.636 & 0.851 & 0.965 & 9 & 0.742 & 0.932 & 0.994 \\
\hline 1642 & 135 & 137 & 0.956 & 0.980 & 0.992 & 168 & 0.752 & 0.800 & 0.843 & 106 & 0.956 & 0.980 & 0.992 \\
\hline 1645 & 99 & 171 & 0.521 & 0.576 & 0.633 & 167 & 0.533 & 0.590 & 0.647 & 99 & 0.972 & 0.992 & 0.998 \\
\hline 1648 & 0 & 0 & N/A & $\mathrm{N} / \mathrm{A}$ & $\mathrm{N} / \mathrm{A}$ & 0 & $\mathrm{~N} / \mathrm{A}$ & $\mathrm{N} / \mathrm{A}$ & $\mathrm{N} / \mathrm{A}$ & 0 & N/A & N/A & $\mathrm{N} / \mathrm{A}$ \\
\hline 1651 & 2 & 20 & 0.039 & 0.123 & 0.267 & 20 & 0.039 & 0.123 & 0.267 & 3 & 0.248 & 0.613 & 0.900 \\
\hline 1654 & 31 & 201 & 0.120 & 0.154 & 0.195 & 355 & 0.068 & 0.087 & 0.109 & 33 & 0.827 & 0.921 & 0.974 \\
\hline 1657 & 17 & 170 & 0.070 & 0.100 & 0.140 & 52 & 0.234 & 0.329 & 0.437 & 27 & 0.471 & 0.622 & 0.759 \\
\hline 1660 & 66 & 499 & 0.114 & 0.131 & 0.151 & 501 & 0.114 & 0.131 & 0.150 & 130 & 0.439 & 0.506 & 0.573 \\
\hline 1663 & 0 & 168 & 0 & 0.003 & 0.015 & 0 & N/A & N/A & N/A & 4 & 0.010 & 0.128 & 0.448 \\
\hline 1666 & 1 & 320 & 0.001 & 0.003 & 0.011 & 1 & 0.223 & 0.706 & 0.974 & 48 & 0.006 & 0.032 & 0.089 \\
\hline 1669 & 0 & 541 & 0 & 0 & 0.003 & 0 & N/A & N/A & N/A & 109 & 0 & 0.005 & 0.024 \\
\hline 1672 & 47 & 625 & 0.064 & 0.074 & 0.086 & 459 & 0.086 & 0.101 & 0.121 & 262 & 0.148 & 0.179 & 0.215 \\
\hline 1675 & 47 & 561 & 0.070 & 0.082 & 0.097 & 196 & 0.197 & 0.239 & 0.287 & 108 & 0.363 & 0.434 & 0.509 \\
\hline 1678 & 30 & 538 & 0.045 & 0.055 & 0.068 & 415 & 0.057 & 0.071 & 0.089 & 121 & 0.193 & 0.248 & 0.312 \\
\hline 1681 & 30 & 516 & 0.047 & 0.057 & 0.070 & 738 & 0.034 & 0.039 & 0.047 & 146 & 0.159 & 0.205 & 0.260 \\
\hline 1684 & 72 & 683 & 0.093 & 0.104 & 0.116 & 264 & 0.235 & 0.272 & 0.312 & 186 & 0.334 & 0.386 & 0.440 \\
\hline 1687 & 42 & 697 & 0.051 & 0.058 & 0.068 & 432 & 0.080 & 0.096 & 0.116 & 190 & 0.179 & 0.221 & 0.268 \\
\hline 1690 & 12 & 839 & 0.011 & 0.013 & 0.016 & 362 & 0.022 & 0.032 & 0.047 & 374 & 0.022 & 0.031 & 0.046 \\
\hline 1693 & 0 & 786 & 0 & U & 0.001 & 0 & N/A & N/A & N/A & 298 & 0 & 0.001 & 0.007 \\
\hline 1696 & 4 & 944 & 0.004 & 0.004 & 0.005 & 311 & 0.006 & 0.013 & 0.025 & 534 & 0.004 & 0.006 & 0.012 \\
\hline 1699 & 9 & 945 & 0.008 & 0.008 & 0.010 & 198 & 0.028 & 0.046 & 0.072 & 528 & 0.011 & 0.016 & 0.024 \\
\hline 1702 & 122 & 935 & 0.123 & 0.129 & 0.136 & 752 & 0.150 & 0.161 & 0.174 & 535 & 0.206 & 0.226 & 0.248 \\
\hline 1705 & 332 & 883 & 0.363 & 0.374 & 0.386 & 880 & 0.364 & 0.375 & 0.387 & 511 & 0.623 & 0.647 & 0.673 \\
\hline 1708 & 252 & 852 & 0.283 & 0.294 & 0.306 & 903 & 0.268 & 0.278 & 0.288 & 450 & 0.530 & 0.558 & 0.587 \\
\hline 1711 & 6 & 725 & 0.005 & 0.006 & 0.011 & 257 & 0.013 & 0.023 & 0.040 & 139 & 0.024 & 0.045 & 0.078 \\
\hline 1714 & 128 & 698 & 0.169 & 0.182 & 0.196 & 631 & 0.185 & 0.201 & 0.219 & 160 & 0.746 & 0.796 & 0.841 \\
\hline
\end{tabular}

Notes. Columns are: \#1 - central year of the triennial interval; \#2 - number of active days $N_{\mathrm{A}}$ within the interval; \#3, 7 and 11 - number of total observational days $N_{\mathrm{T}}$ considered in the three models, respectively; \#4, 8 and 12 - lower $90 \%$ bound of the active day fraction, for the tree models, respectively; \#5, 9 and 13 - median active day fraction, for the tree models, respectively; \#6, 10 and 14 - upper 90\% bound of the active day fraction, for the tree models, respectively.

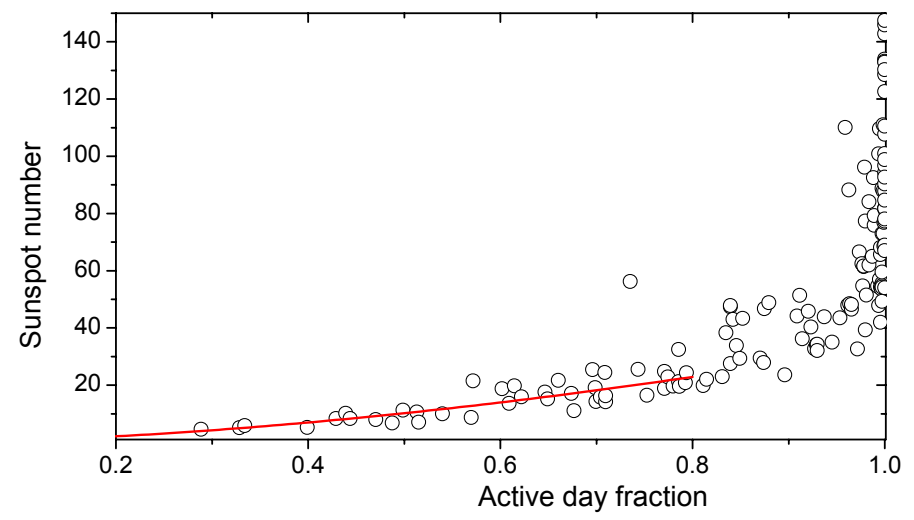

Fig. 5. Relation between triennial sunspot numbers and active-day fraction for the period 1850-1995 using the group sunspot number (Hoyt \& Schatten 1998a). The red curve is the best fit relation $R=33.6 \cdot F_{A}^{1.72}$.

corresponded to the growth phase of the highest solar cycle \#19) and can be approximated well by a dependence $R=33.6 \cdot F_{A}^{1.72}$. The relation loosens for $F_{A}>0.8$ and is lost completely with the active-day fraction approaching unity. Thus, the active-day fraction is a good index of sunspot activity until it reaches 0.8 .

Using these dependencies, we have evaluated the sunspot numbers during the period analysed, as shown in Fig. 6. One can see that the sunspot numbers appear below two during the deep MM (1645-1700) and seven around 1705 in the least conservative model ML. The optimum MO model yields the sunspot number not exceeding five for the deep MM and seven around
1705 (except for the very uncertain period around 1666 with the lack of observations). The most conservative MS model yields sunspot cycles below ten during the core MM and a possible relatively high cycle in the 1650 s, which is based on the lack of overlapping records from different observers, and about $15 \mathrm{ca} .1705$. Even this very strict model suggests that the cycles were lower than 15-20 in sunspot numbers, which is much lower than the present cycle \#24 and an order of magnitude lower than the very high cycles proposed by Zolotova \& Ponyavin (2015). Considering the severe reduction of the statistics and a possible strong bias towards active days in the MS model, we believe it is not indicative of the true solar activity evolution during the MM and may represent only the uppermost upper (maximum maximorum) bound.

\section{Conclusions}

Using three models of different levels of conservatism to treat generic "no-sunspot" statements, we have created a database of reliable sunspot observation around the Maunder minimum (1637-1715) and revised the sunspot cyclic activity over that period. We show that:

1. A large number of no-spot records, corresponding to the solar meridian observations, may be unreliable in the HS98 database.

2. The active-day fraction remained low (below 0.3-0.4) throughout the MM, indicating the low level of sunspot activity. 


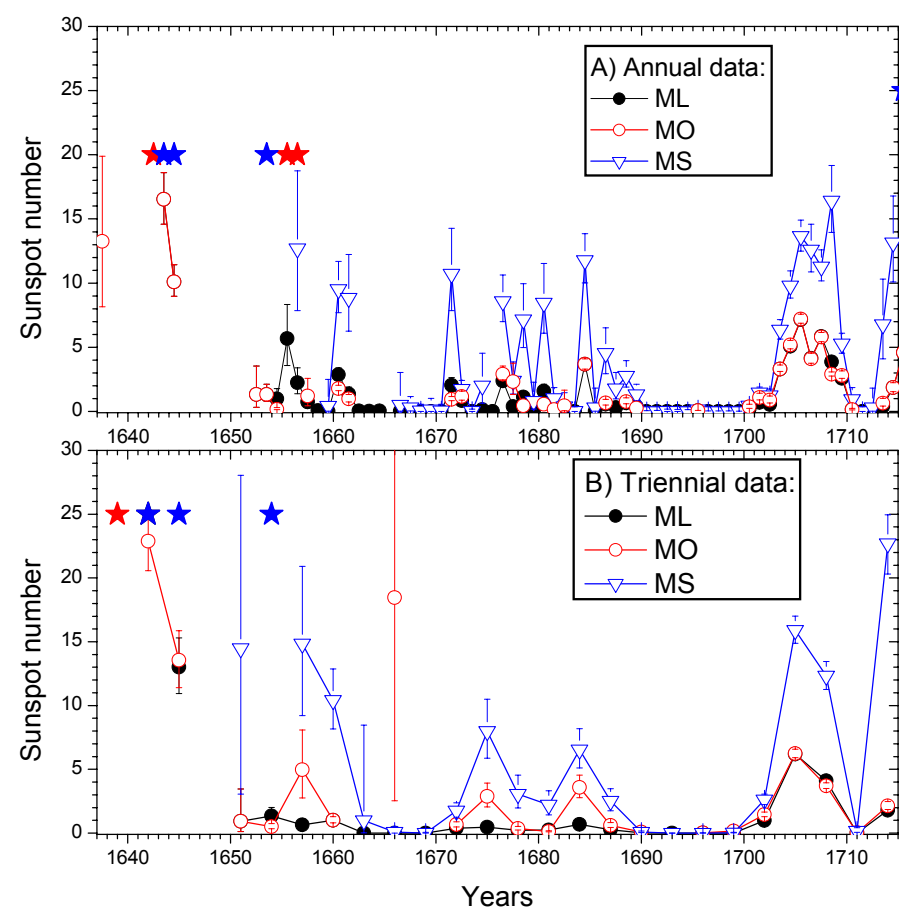

Fig. 6. Annual (panel A)) and triennial (panel B)) sunspot numbers reconstructed in the three models as denoted in the legends. Years with low statistics $\left(N_{\mathrm{T}}<10\right)$ are not shown. Stars indicate that the sunspot number cannot be assessed from the active-day fraction (see text) and is greater than $18 / 23$ for the annual/triennial data.

3. The solar cycle appears clearly during the core MM with maxima at 1657, 1675, 1684, 1705, and possibly 1666 .

4. The length of the solar cycle during the MM appears shorter ( $9 \pm 1$ years) than the standard 11-year solar cycle, but there is an uncertainty in that. $\mathrm{A} \approx 20$-year clustering of activity is also observed.

5. The magnitude of the sunspot cycle during MM is assessed to be below five (10 in the most conservative model) in sunspot numbers. The exact level is hardly possible to determine, but it is below ten.

6. High solar cycles during the MM, as proposed by Zolotova \& Ponyavin (2015), contradict the data.

We note that this is an uppermost upper (maximum maximorum) bound for solar activity during MM because of a possible selection bias (particularly important in the MS model), and the true level of activity may be less than that.

Only a thorough review of each record and each solar observation during the MM can make it possible to reveal the best picture of solar activity during this period. Therefore, we encourage researchers (especially Latin scholars) to query and analyse the old texts to understand how the observations were made and the true level of solar activity they indicate.

Acknowledgements. Support from the Junta de Extremadura (Research Group Grant No. GR10131), from the Ministerio de Economía y Competitividad of the Spanish Government (AYA2011-25945 and AYA2014-57556-P), and from the COST Action ES1005 TOSCA (http://www.tosca-cost.eu) is gratefully acknowledged. I.U. and G.K. acknowledge support from the ReSoLVE Centre of Excellence (Academy of Finland, project no. 272157). G.K. acknowledges partial support from Programme No. 9 of the Presidium RAS.

\section{References}

Beer, J., Tobias, S., \& Weiss, N. 1998, Sol. Phys., 181, 237

Carrasco, V. M. S., Villalba Álvarez, J., \& Vaquero, J. M. 2015, Sol. Phys., submitted, ArXiv e-prints [arXiv: 1502 . 06270]

Casas, R., Vaquero, J. M., \& Vazquez, M. 2006, Sol. Phys., 234, 379

Clette, F., Svalgaard, L., Vaquero, J. M., \& Cliver, E. 2014, Space Sci. Rev., 186, 35

Eddy, J. 1976, Science, 192, 1189

Hoyt, D. V., \& Schatten, K. H. 1998a, Sol. Phys., 179, 189

Hoyt, D. V., \& Schatten, K. H. 1998b, Sol. Phys., 181, 491

Kovaltsov, G. A., Usoskin, I. G., \& Mursula, K. 2004, Sol. Phys., 224, 95

Manfredi, E. 1736, De Gnomone Meridiano Bononiensi ad Divi Petronii (Bononiae: Laeli a Vulpa), 397

Mendoza, B. 1997, Annales Geophys., 15, 397

Miyahara, H., Masuda, K., Muraki, Y., et al. 2004, Sol. Phys., 224, 317

Miyahara, H., Sokoloff, D., \& Usoskin, I. 2006, in Solar Terrestrial (ST), eds.

W.-H. Ip, \& M. Duldig (Singapore; Hackensack, USA: World Scientific), Adv. Geosciences, 2, 1

Miyahara, H., Kitazawa, K., Nagaya, K., et al. 2010, J. Cosmol., 8, 1970

Miyake, F., Masuda, K., \& Nakamura, T. 2013, Nature Comm., 4, 1748

Nagaya, K., Kitazawa, K., Miyake, F., et al. 2012, Sol. Phys., 280, 223

Owens, M. J., Usoskin, I., \& Lockwood, M. 2012, Geophys. Res. Lett., 39, L19102

Ribes, J., \& Nesme-Ribes, E. 1993, A\&A, 276, 549

Roth, R., \& Joos, F. 2013, Clim. Past, 9, 1879

Soon, W.-H., \& Yaskell, S. 2003, The Maunder Minimum and the Variable SunEarth Connection (Singapore; River Edge, USA: World Scientific)

Stuiver, M., Reimer, P., Bard, E., et al. 1998, Radiocarbon, 40, 1041

Usoskin, I. G. 2013, Liv. Rev. Solar Phys., 10, 1

Usoskin, I., Mursula, K., \& Kovaltsov, G. 2001, J. Geophys. Res., 106, 16039

Vaquero, J. M. 2007, Adv. Space Res., 40, 929

Vaquero, J. M., \& Trigo, R. M. 2014, Sol. Phys., 289, 803

Vaquero, J. M., \& Vázquez, M. 2009, The Sun Recorded Through History: Scientific Data Extracted from Historical Documents (Berlin: Springer), Astrophys. Space Sci. Lib., 361

Vaquero, J. M., Gallego, M. C., Usoskin, I. G., \& Kovaltsov, G. A. 2011, ApJ, 731, L24

Vaquero, J. M., Trigo, R. M., \& Gallego, M. C. 2012, Sol. Phys., 277, 389

Vaquero, J. M., Gutiérrez-López, S., \& Szelecka, A. 2014, Adv. Space Res., 53, 1180

Vaquero, J. M., Nogales, J. M., \& Sánchez-Bajo, F. 2015, Adv. Space Res., 55, 1546

Waldmeier, M. 1961, The Sunspot Activity in the Years 1610-1960 (Zürich: Zurich Schulthess and Company AG)

Zolotova, N. V., \& Ponyavin, D. I. 2015, ApJ, 800, 42 